\title{
Modeling and simulation of piezoelectric generator with indirect contact excited by vortex-induced vibration
}

\author{
Hajar Mazar ${ }^{1 *}$, Bouchra Rzine ${ }^{1}$, Saida Bahsine $^{2}$, Mohammed Radouani ${ }^{3}$, Benaissa Elfahime ${ }^{3}$, and M'hammed El \\ Gadari $^{3}$ \\ ${ }^{1}$ LGM, Faculté des Sciences et Techniques de Fès, Université Sidi Mohamed Ben Abdellah, Maroc. \\ hajar.mazar2@usmba.ac.ma , bouchra.rzine@usmba.ac.ma . \\ ${ }^{2}$ LPFAS, Faculté Poly-Disciplinaire Safi, Université Cadi Ayyad, Maroc. \\ saida.bahsine@yahoo.com \\ ${ }^{3}$ IMSM, Ecole Nationale Supérieure des Arts et Métiers de Meknès, Université Moulay Ismaïl, Maroc. \\ m.radouani@ensam-umi.ac.ma b.elfahime@ensam-umi.ac.ma, m.elgadari@ensam-umi.ac.ma.
}

\begin{abstract}
In the last years, the new research papers piezoelectric energy harvesting from vortex-induced vibration (PEH-VIV) are focused on indirect contact between airflow and piezoelectric transducer. Where some researchers have noticed that is difficult to establish an accurate analytical model that could describe precisely the behavior of the piezoelectric generator including the indirect interaction with airflow. Due to these difficulties, new studies are concentrating directly on the experimental investigations to get results of energy harvesters of the indirect-contact piezoelectric wind energy harvesters (IC-PWEHs). However, this paper presents an analytical model that can provide good results according to experimental tests. The obtained results concern the displacement of the piezoelectric transducer indirectly excited by a vortexinduced vibration (VIV) and the generated voltage according to a different mass of cylindrical shell. The numerical results of the model show a significant agreement with the experimental results due to the good estimation of the system's parameters, where the computing is done numerically using the MATLAB Simulink. Additionally, the optimal mass value of the piezoelectric transducer was determined approximately by the proposed analytical model. Where this mass is equivalent to the highest voltage amplitude according to the experimental tests.
\end{abstract}

\section{Introduction}

Recently, renewable energy harvesting technology has been promoted due to its importance in resolving the higher consumption of non-renewable energy, the matter of energy shortage, and pollution. Over the past 20 years, wireless sensor networks (WSNs) are widely used and distributed within the world. Designing suitable energy harvesters to power WSNs has attracted attention from researchers. Three mechanisms could be used to convert ambient vibration energy into electric energy considering piezoelectricity, electrostatic induction, and the electromagnetic induction [1].

Piezoelectric energy harvesting is pointed due to its high voltage and low current, making it suitable to be used in low-power electrical stuff, especially within the field of WSNs [2-4]. In the late 1990s, piezoelectric energy harvesting technology by vibration was widely used to harvest energy from the environmental flow and convert it into vibrational energy [5].

In recent years, many meaningful research works have been carried out on using vortex-induced vibration to collect ocean energy and wind energy. In addition, advanced nonlinear techniques including an intermediate element are employed to improve energy harvesting performance $[2,5]$. The old works specialize in the direct interaction between the structure (piezoelectric cantilevers) and airflow during a variety of speeds between 1 and $10 \mathrm{~m} / \mathrm{s}$ [6-12], while within the newest works the interaction is indirect by adding an intermediate. Recently an experimental work offers an alternate solution to beat the deficiency caused by the direct interaction. The flow energy harvesting is based on the vortex-induced vibrations (VIV) of a piezoelectric transducer integrated into a cylinder, the power generated from the wind speed range $[0-40 \mathrm{~m} / \mathrm{s}]$ is about $2.87 \mathrm{~mW}$ [13]. The non-contact interaction helps to avoid deformation and rupture of piezoelectric transducer because of resonance frequencies and to reinforce reliability and adaptableness to the environment. This work is completed just experimentally, focused on studying the feasibility and environmental adaptability through experiments, to reinforce the performance and applicability of power harvesting. Among the advantages of the thought of indirect-contact wind energy harvesting is that the straightforward adapting the dynamic systems to different wind speeds by adjusting structural parameters, just like the mass of the intermediate (cylinder shell) and thus the mass of the transducer.

Several approaches have been employed in the literature to control or suppress vibration in engineering structures or benefit from these vibrations to generate electricity [13-15]. Moreover, reducing or controlling VIV in parallel with energy harvesting is desired for enhancing the safety of structures, performance, and lifetime.

In this paper, the investigation aims to get an analytical model that could give the same results of the experiments ones of the article of Kan and other authors [13] by using numerical simulation, The results of the proposed analytical model are compared to the experiments ones. A detailed system's parameters are 
evaluated to determine their effects on the behaviors of output parameters displacement and voltage. The rest of this article is organized as follows. Section 2, the analytical model of NC-PWEH is briefly presented with the parameter estimates. Section 3 contains the comparison of the responses of the cylinder shell and piezoelectric transducer obtained for different masses with previous experiments done by Kan and their partners [13]. Some conclusions are presented in section 4.

\section{Mathematical model and key factor estimation}

\subsection{Mathematical model}

The main objective of this paper is to seek out an appropriate analytical model of the system of [13]. The system is summed up during a piezoelectric transducer made from two cantilevered piezoelectric beams, one up and the other down, with a particular bending curvature and copper sheet, and its fixed end is installed within the cylinder to which the added cantilever mass exists at the free end of the transducer, and for the cylinder, especially on its inner wall, another mass is attached. Its outer ends are fixed to the support by four springs. Therefore, the cylindrical shell is suspended horizontally and located within the direction of the incoming airflow [13]. Fig. 1 shows an approximate representation of this technique by the spring-massdamper model. Which $M_{1}$ represents the equivalent mass of the cylindrical shell, $K_{1}$ represents the equivalent stiffness of 4 linear springs which are fixed in two supports $\left(S_{1}, S_{2}\right)$ and $C_{1}$ represents the viscous damping coefficient of the cylinder. The cylindrical shell is connected to the bottom of the piezoelectric transducer, $M_{2}$, via a linear spring element, $K_{2}$, also as a linear viscous damping element of coefficient $C_{2}$. The force $\mathrm{F} 1$ represents the external excitation of the fluid flow on the cylinder, and $Y_{1}, Y_{2}$ represent the transverse displacement of the cylinder and therefore the displacement of the piezoelectric transducer, respectively. Also constrained to be perpendicular to the airflow.

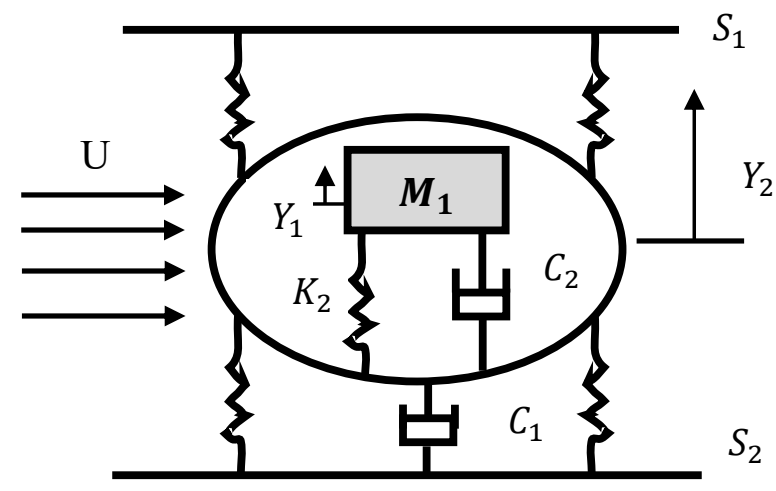

Fig. 1. Model of a coupled system of the NC-PWEHs.

The piezoelectric transducer considered is of the PZT-4 type, the same as that studied previously in the article by [13]. It connects to a resistor R, and it plays the role of a capacitor. $\theta$ is the electromechanical coefficient relating to the material property of the piezoelectric element and the structural dimension. $\mathrm{V}$ represents the output voltage of the transducer. Using Newton's method, the equations governing the motion of the energy sensor are often formulated and are given by

$M_{1} \ddot{Y}_{1}+C_{1} \dot{Y}_{1}+K_{1} Y_{1}+C_{2}\left(\dot{Y}_{1}-\dot{Y}_{2}\right)+K_{2}\left(Y_{1}-Y_{2}\right)=$
$F l$

$M_{2} \ddot{Y}_{2}+C_{2}\left(\dot{Y}_{2}-\dot{Y}_{1}\right)+K_{2}\left(Y_{2}-Y_{1}\right)=0$

$V=\frac{K_{2} Y_{2}}{\theta}$

By defining

$\omega=\sqrt{\frac{K_{t}}{M_{t}}}, \quad M_{t}=M_{1}+M_{2}, \quad K_{t}=\frac{K_{1} K_{2}}{K_{1}+K_{2}}$,

$F_{\text {lift }}=F l=\frac{1}{2} \rho_{\text {air }} U^{2} H C_{L} \cos \left[\omega_{V I V} t\right], \quad \omega_{V I V}=\frac{2 \pi U S_{t}}{D}$

Where $K_{t}, M_{t}$ are the equivalent stiffness and mass, $\omega$ is the frequency of the coupled system, and $\theta$ is the electromechanical coupling coefficient. $F_{\text {lift }}$ is the vortex-induced vibration force, $\omega_{V I V}$ is the vortex shedding frequency for the VIV. $\rho_{\text {air }}$ is the air density, $\mathrm{D}$ and $\mathrm{H}$ are the diameter and the height of the cylinder, $\mathrm{U}$ is the wind speed, $S_{t}$ is the Strouhal number, and $C_{L}$ is the lift coefficient of the cylinder.

\subsection{Key factor estimation}

The exact values for some parameters are not available. Therefore, in the course of this work, some estimations are made with the aim that the results of this model that we made are as close as possible to the experimental results.

\subsubsection{Estimation of the Strouhal number (St)}

Vortex shedding happens when the wind hits a structure, causing alternating vortices to form at a certain frequency. This in turn causes the system to excite and produce a vibrational load. The various vortex shedding processes, occurring with increasing Reynolds number, are described by different coefficients St (Strouhal number). The vortex shedding frequency is calculated using the Reynolds number (which describes the fluid flow characteristics) and the Strouhal number (which describes the oscillations of a fluid). The Reynolds number is calculated using viscosity, density, flow velocity, and some geometry from the object in the fluid. It is calculated over a range of flow speeds. The Strouhal number is then calculated from those Reynolds numbers.

The Strouhal Number has a crucial influence on the frequency at which vortex shedding occurs, it is commonly used to measure the wake vortex shedding after a bluff-body. Again, the Strouhal Number is dependent on Reynolds Number [16]. The relation between these two dimensionless numbers can be represented by the graph obtained from MIT OCW. Data is taken from Lienhard (1966) and Achenbach and 
Heinecke (1981). Which we can note that the Strouhal Number remains nearly constant at approximately 0.2 unrelated to the geometry of any bluff body over a broad range of Reynolds Numbers [16].

The Strouhal number depends on the Reynolds number which also depends on the wind speed. Thus, the first step is to determine the Reynolds number corresponding to the interval of the wind speed [5-40 $\mathrm{m} / \mathrm{s}$, then the values of Strouhal numbers in the range of $22.43 \times 10^{3} \sim 179.49 \times 10^{3}$ of Reynolds numbers could be estimated by the diagram of the relationship between Strouhal number and Reynolds number for circular cylinders [17]. Fig. 2. Shows the plot of the Strouhal number consistent with the wind speed.

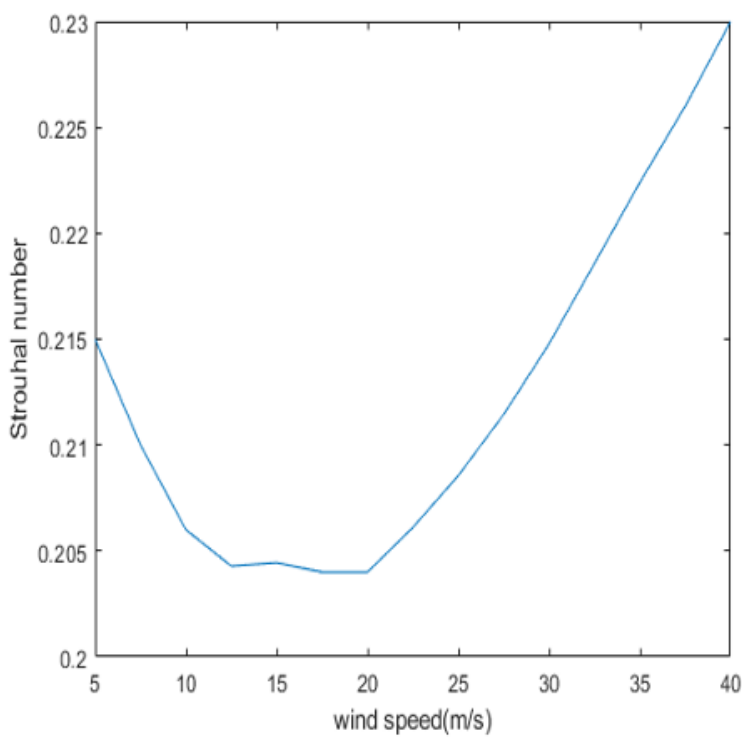

Fig. 2. Variation of Strouhal numbers of the cylinder with different wind speed

The function of the Strouhal numbers decreases in the range of 5 to $10 \mathrm{~m} / \mathrm{s}$, and it is almost constant with a variation of the wind between 10 and $20 \mathrm{~m} / \mathrm{s}$, then it increases with a linear form ranging from a wind speed equal to $20 \mathrm{~m} / \mathrm{s}$. The rest of this article will show that this variation of the values of the Strouhal number can require an influence on the output of the analytical model, in the level of the form of the oscillation of airflow.

\subsubsection{Estimation of the lift coefficient $\left(C_{L}\right)$}

To define the numerical value of coefficient lift $C_{L}$. We study our system Fig. 1 in equilibrium state as shown in Fig. 3 with constant airspeed to be able to use the law of inertia: Lift=Weight

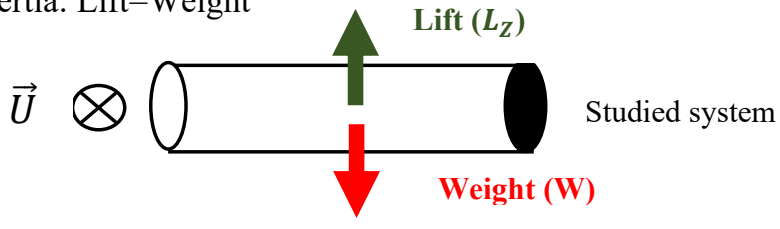

Fig. 3. Cylindrical shell and piezoelectric transducer form a system that is acted on by external forces. The weight $W$ and the lift $\boldsymbol{L}_{\boldsymbol{Z}}$ from the airflow are two external forces acting on the system.
Where weight $\mathrm{W}$ is the equivalent mass $M_{t}$ of the system multiplied by gravitational acceleration $\mathrm{g}$. The next force is an aerodynamic force which is lift $L_{Z}$. It was experimentally determined that lift depend on dynamic pressure $\mathrm{q}$ which is equal to one-half density $1 / 2 \rho$ times velocity squared $U^{2}$, area of the wind $\mathrm{S}$, and the lift coefficient $C_{L}$ which depends on the system's shape and angle of attack.

$$
\begin{aligned}
W & =L_{Z} \\
M_{t} g & =q \mathrm{~S} C_{L} \\
M_{t} g & =1 / 2 \rho U^{2} \mathrm{~S} C_{L}
\end{aligned}
$$

From eq. (6) we can determine the numerical value of coefficient lift $C_{L}$

$$
C_{L}=\frac{\rho U^{2} S}{2 M_{t} g}
$$

The lift coefficient is proportional to velocity squared. In other word lift coefficient increase with the increase of the wind speed. For the range of wind speed [5-40 m/s] we get a range of lift coefficient between 0.3 and 0.6. On another side, some studies find that changing $C_{L}$ doesn't affect the output curves shape of voltage. Although fluctuating lift coefficient $C_{L}$ only influences the hump area for the VIV-based wind energy harvester with cylinder, and other studies give the values of the aerodynamic coefficients for typical cases among them the lift coefficient of the cylinder which is 0.3 $[18,11]$. Thus, our calculation, previous studies, and the angle of attack which is near to zero. All of them prove that 0.3 is a suitable value to lift coefficient in this work.

\subsubsection{Estimation of other parameters}

To make the model more accurate, some other proprieties of our system should be added to approach the exact values of the parameters below.

$$
\left\{\begin{array}{l}
\xi=\frac{\ln (\delta)}{\sqrt{4 \pi^{2}+\ln (\delta)^{2}}} \\
C_{1}=2 M_{t} w \xi \\
C_{2}=\gamma C_{1} \\
C_{p}=\frac{\epsilon_{33} b l_{p}}{h_{p}} \\
\theta=\sqrt{\beta M_{2} C_{p}}
\end{array}\right.
$$

Where $M_{t}$ is the equivalent mass of a generator piezoelectric, $\mathrm{C}$ is the damping related to the damping coefficient $\xi, \gamma=0.8$ is the ratio of damping. $\Theta$ is the electromechanical coupling coefficient, $C_{p}$ is the internal capacitance, $l_{p}, k_{p}, h_{p}$ are respectively length, width, and thickness of piezoelectric element PZT-4, $\epsilon_{33}=54.7 \times 10^{-10} \mathrm{~F} / \mathrm{m}$ is the permittivity [19], $\beta=$ $94.92 \times 10^{3}(\mathrm{rad} / \mathrm{s})^{2}$ is the natural frequency of the system that can be obtained by the free decay experiment, and $\delta=1.007$ is the ratio of the amplitude before and after the free decay experiment [2]. 


\section{Results and discussions}

As mentioned in section 2, the upper and lower cantilevered piezoelectric plates in different wind speeds (U) were very similar, and they excite simultaneously to generate a voltage $(\mathrm{Vg})$ because of their symmetrical configuration. In another word, the relationships between the generated voltage and airflow from the upper and lower piezoelectric plates were the same. So, one piezoelectric plate was used to evaluate the output parameters of the IC-PWEHs in this study. In this direction, the influence of the below estimation parameters on the vibration behaviors will be studied through simulation. In this section, we will study numerically the relationship between the wind speed and the vibration displacement/generated voltage with different cylindrical shell mass and piezoelectric transducer mass.

To verify the feasibility of the model, to show the influence of the mass of the cylindrical shell, the mass of the piezoelectric transducer, and the wind speed on the output parameters of the NC-PWEH. The geometric parameters of the cylinder and piezoelectric, and the other estimate value of equations $(8,9,10,11,12)$ in Table 1 , which are used in subsequent studies:

Table 1. parameters of cylinder and piezoelectric.

\begin{tabular}{|l|c|c|c|}
\hline PARAMETER & SYMbOL & VALUE & UNIT \\
\hline $\begin{array}{l}\text { THE EQUIVALENT } \\
\text { STIFFNESS OF SPRING }\end{array}$ & $K_{1}$ & 260 & $\mathrm{~N} / \mathrm{m}$ \\
\hline AIR DENSITY & $\rho_{\text {air }}$ & 1.255 & $\mathrm{Kg} / \mathrm{m}^{-3}$ \\
\hline $\begin{array}{l}\text { LENGTH OF } \\
\text { CYLINDRICAL SHELL }\end{array}$ & $\mathrm{H}$ & 160 & $\mathrm{~mm}$ \\
\hline
\end{tabular}

\begin{tabular}{|l|c|c|c|}
\hline LOAD RESISTANCE & $\mathrm{R}$ & 300 & $\mathrm{~K} \Omega$ \\
\hline $\begin{array}{l}\text { DIAMETER OF } \\
\text { CYLINDRICAL SHELL }\end{array}$ & $\mathrm{D}$ & 70 & $\mathrm{~mm}$ \\
\hline $\begin{array}{l}\text { WIDTH OF } \\
\text { PIEZOELECTRIC PZT-4 }\end{array}$ & $k_{p}$ & 40 & $\mathrm{~mm}$ \\
\hline $\begin{array}{l}\text { THICKNESS OF } \\
\text { PIEZOELECTRIC PZT-4 }\end{array}$ & $h_{p}$ & 0.2 & $\mathrm{~mm}$ \\
\hline
\end{tabular}

Table 2. Estimation of some parameters.

\begin{tabular}{|l|c|c|}
\hline PARAMETER & SYMBOL & VALUE \\
\hline COEFFICIENT LIFT & $C_{L}$ & 0.3 \\
\hline $\begin{array}{l}\text { LENGTH OF CYLINDRICAL } \\
\text { SHELL }\end{array}$ & $\theta$ & 0.014 \\
\hline DAMPING COEFFICIENT & $\xi$ & 0.001 \\
\hline INTERNAL CAPACITANCE (F) & $C_{p}$ & $3.87 \times 10^{-8}$ \\
\hline
\end{tabular}

\subsection{The effect of cylindrical shell mass on output parameters: displacement and voltage}

For the different simulations, $M_{2}=55 \mathrm{~g}$ is considered as the standard mass of the piezoelectric as done in [13]. the subsequent study tested the influence of the mass of wind speed in the range of $[5-40 \mathrm{~m} / \mathrm{s}]$. To validate the analytical model, it is necessary to compare the results of our numerical simulations with the experimental results of [13]. For that purpose, some points of the voltage experiment curves were taken and plotted by the MATLAB software (red stars) to note how close the two curves are. Fig.4 illustrates the comparison between the analytical model and the experimental results in the case of $\mathrm{M} 1=160 \mathrm{~g}$.

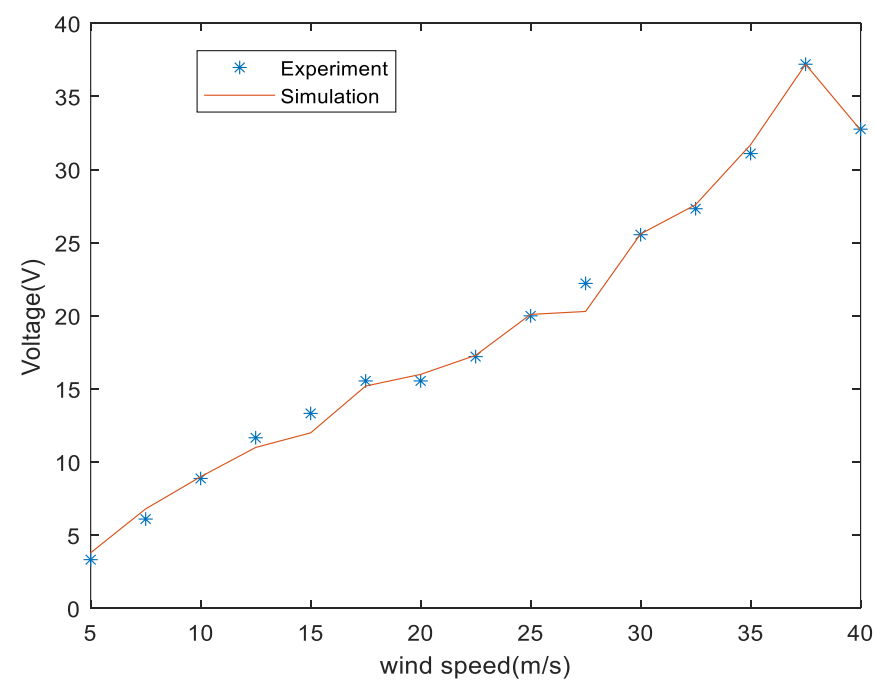

Fig. 4. Performance comparison between the analytical model and experimental results of [13] in the case of voltage output for $\boldsymbol{M}_{\mathbf{1}}=160 \mathrm{~g}$

Fig. 4 shows a fairly similar trend in response curves of the generated voltage (V) versus wind speed (U) for the IC-PWEHs under a mass of cylindrical shell M1 $=160 \mathrm{~g}$. Nevertheless, the inclines of the relationship curves between $\mathrm{V}$ and $\mathrm{U}$ are slightly different between simulation (S) and experiment (E). Some values of S/E curves under cylindrical shell mass $160 \mathrm{~g}$ demonstrate this difference: the generated voltages of $11 / 11.665 \mathrm{~V}$, $12 / 13.33 \mathrm{~V}$ and $20.3 / 22.22 \mathrm{~V}$ were measured at 12.5 $\mathrm{m} / \mathrm{s}, 15 \mathrm{~m} / \mathrm{s}$ and $27.5 \mathrm{~m} / \mathrm{s}$, respectively. Also note, the generated voltages show an increasing trend as the wind speed increases. The maximum generated voltage (peak 
voltage) of $37.2 \mathrm{~V}$ was observed when the wind speed was increased to $37.5 \mathrm{~m} / \mathrm{s}$, for the IC-PWEHs under the cylindrical shell mass of $160 \mathrm{~g}$. It meant that there is an optimum wind speed about $37 \mathrm{~m} / \mathrm{s}$ to obtain the maximum generated voltage of the cylindrical shell. In sum, the variation of the results of the experiment coincides with the curve of the proposed analytical devices and the proposed model successfully prophesies the full trend of the response curve of the voltage output experiment. An equivalent observation for the other mass $275 \mathrm{~g}$ and $390 \mathrm{~g}$ [13].

The following Fig.5 shows the variation of the displacement of the cylindrical shell according to wind speed under different mass $(160 \mathrm{~g}, 275 \mathrm{~g}, 390 \mathrm{~g})$.

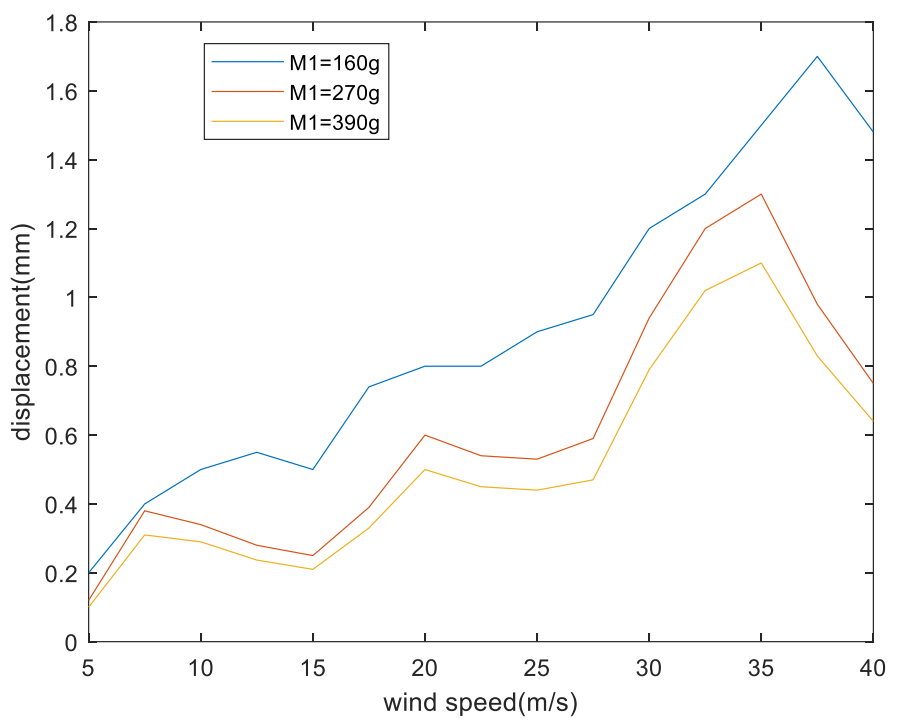

Fig. 5. Variation displacement of the cylindrical shell according to wind speed under different mass

Fig. 5 illustrates the vibration displacement of the cylindrical shell versus wind speed for the IC-PWEHs under different mass of cylindrical shell (M1). The analytical results depict that in the range of wind speed $[5-40 \mathrm{~m} / \mathrm{s}]$. The displacement of cylindrical shell shows an increasing trend as the wind speed increases. And the vibration displacement demonstrates an increasing trend as the wind speed decreases like what was found by the experience of [13]. The maximum vibration displacement of cylindrical shell was increased from 1.1 $\mathrm{mm}$ to $1.7 \mathrm{~mm}$ with the decreasing shell mass from 390 $\mathrm{g}$ to $160 \mathrm{~g}$, which were measured at $35 \mathrm{~m} / \mathrm{s}$ and $37.5 \mathrm{~m} / \mathrm{s}$, respectively. Similarly, the displacement presented a rapidly reducing trend at high wind speed about 37.5 $\mathrm{m} / \mathrm{s}$, which was similar to the relationship between the generated voltage and wind speed. In addition, the output vibration displacement and voltage were affected by the mass of cylindrical shell. And there is an optimum wind speed about $37.5 \mathrm{~m} / \mathrm{s}$ and an optimum shell mass about $160 \mathrm{~g}$ to obtain the maximum vibration displacement. In abridgment, the simulated vibration displacement agrees well with the experimental results, which verifies the fidelity of the present mathematical model.

A comparison of the responses of cylindrical shell and piezoelectric is shown in Fig 6.

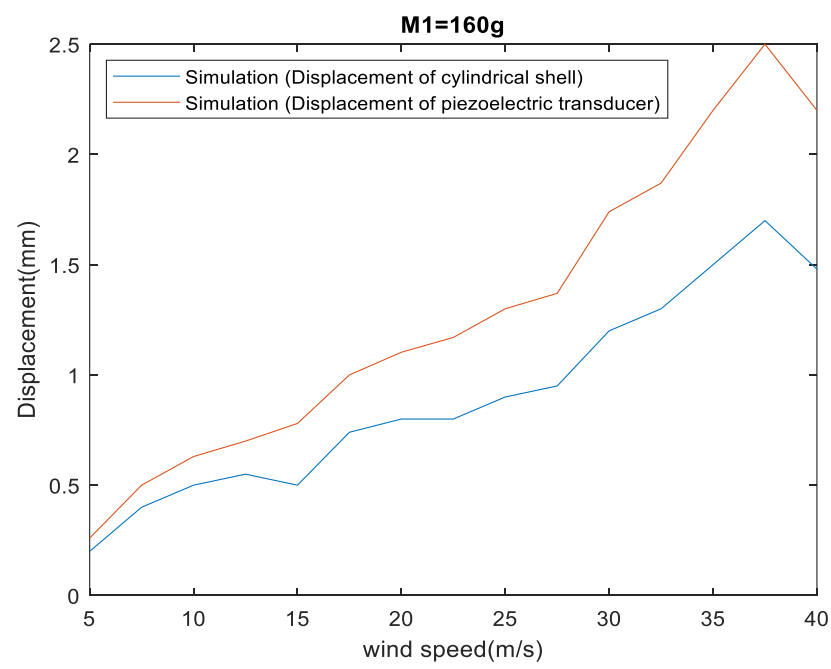

Fig. 6. Performance comparison between the cylindrical shell and piezoelectric transducer of [13] in the event of displacement output for $M_{1}=160 \mathrm{~g}$. 
The oscillation amplitude of the cylindrical shell and piezoelectric transducer grows slowly until wind speed $15 \mathrm{~m} / \mathrm{s}$, but after this value of speed, we observed that the variation of the amplitude of piezoelectric is far exceeding than a cylindrical shell with the increase of wind speed. In another word, the piezoelectric transducer attenuates the oscillation of the cylindrical shell, so it is important to take into account this aspect in a practical implementation such as deciding on the size of the piezoelectric transducer and placing it inside the cylinder.

\subsection{The effect of piezoelectric transducer mass on output parameters}

For this current simulation, $M_{1}=160 \mathrm{~g}$ is considered as the standard mass of the cylindrical shell as done in [13]. Changing the piezoelectric transducer mass $M_{2}$ influence the amplitude and frequency of the piezoelectric transducer, which could indirectly affect the output voltage. Thus, in the following simulation, the effect of the mass of the piezoelectric transducer and the wind speed on the performance of the generation voltage was studied by comparing our analytical results with those of the experiments carried out by [13]. Fig. 7 shows the voltage variation of the NC-PWEH as a function of the wind speed under different masses of the piezoelectric transducer $M_{2}$. The results obtained showed that with the increase in wind speed, the tendency of the tension generated by the height also increased, as shown by experience [13]. The increase in the mass of the piezoelectric shows that there is an optimal mass. for example, the maximum generated voltage of $21.57 \mathrm{~V}$ given by $M_{2}=55 \mathrm{~g}$ with a wind speed of $40 \mathrm{~m} / \mathrm{s}$. For the other values, the height generated voltages of $21.24 \mathrm{~V}$ and $20.91 \mathrm{~V}$ at the same speed of 40 $\mathrm{m} / \mathrm{s}$ under a piezoelectric transducer mass of $45 \mathrm{~g}$ and $65 \mathrm{~g}$, respectively.

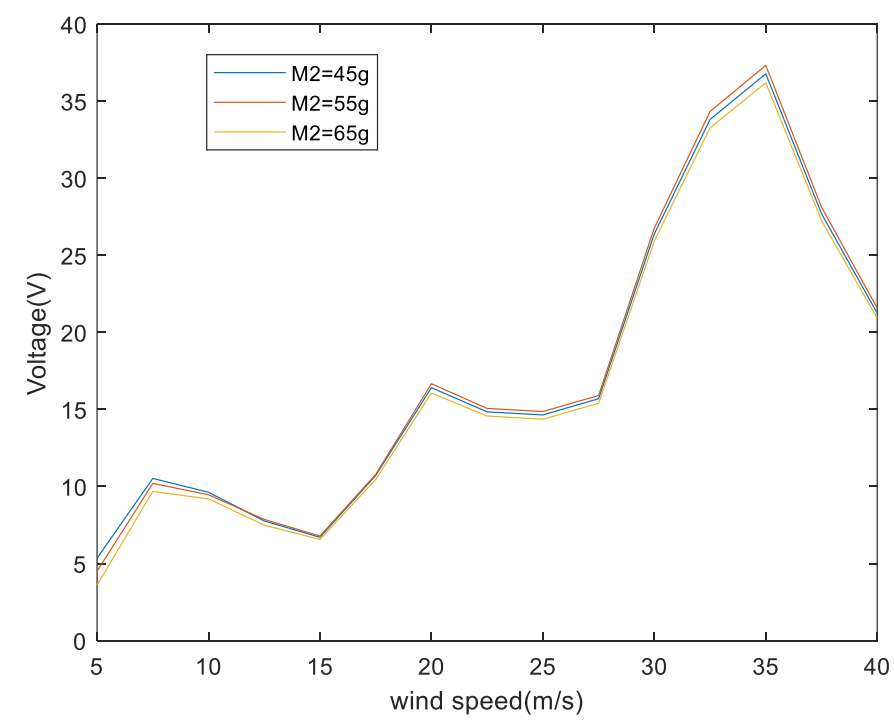

Fig. 7. Generated voltage according to wind speed under a different mass of piezoelectric transducer.

For greater precision, the voltage generated at height gradually increased from $21.24 \mathrm{~V}$ to $21.57 \mathrm{~V}$ with increasing the mass of the transducer from $45 \mathrm{~g}$ to $55 \mathrm{~g}$, then gradually decreased from $21.57 \mathrm{~V}$ to $20.91 \mathrm{~V}$ with increasing transducer mass to $65 \mathrm{~g}$. Our analytical model follows a working rule equivalent to the experimental model which summarizes there the generated voltage increased first, then decreased with the increase in the mass of the piezoelectric transducer. the differences between the models just at the level of the value of the optimal mass. The analytical model is $M_{2}=55 \mathrm{~g}$ but by experience was $M_{2}=65 \mathrm{~g}$ [13].

These results indicated the proximity between the analytical model and therefore the experiment, despite the small deviation from the optimal mass level obtained both from the experiment and from the mathematical model. This discrepancy is often explained by errors in estimates of system parameters and system coupling. More clearly, the optimum mass is influenced by such as the stiffness of the piezoelectric transducer, the
Strouhal number St especially when the beam is thin, and contact between beam and PZT.

\section{Conclusion}

This paper investigates an analytical model to describe the energy harvesting and behavior of indirect contact piezoelectric energy harvester, subjected to the VIV for a wide range of mechanical and electrical excitation levels. Excellent agreement between the model and the experiment is found, due to the proposed estimation of stiffness, damping, lift coefficient, Strouhal number, and electromechanical coupling of the piezoelectric generator.

We explained the slight difference amplitude of $3.5 \%$ by the errors associated with some parameters nonconsidered during this study. However, as concluded in the literature, they can affect the output factors of generator piezoelectric, like the optimal value of 
piezoelectric transducer mass. Finally, we admit that errors in the experiment are inevitable, such as the contact and separation between the beam and the PZT and measurement errors.

\section{References}

[1] J. Wang, S. Zhou, Z. Zhang, and D. Yurchenko. High-performance piezoelectric wind energy harvester with $Y$-shaped attachments. Energy Conversion and Management, 181, 645-652. (2019)

[2] J. Wang, G. Li, M. Zhang, G. Zhao, Z. Jin, K. Xu, and Z. Zhang (2018): Energy harvesting from flowinduced vibration: a lumped parameter model.

[3] S. Zhou, and J. Wang. Dual serial vortex-induced energy harvesting system for enhanced energy harvesting. AIP Advances, 8(7), 075221. (2018)

[4] M. Zhang, G. Zhao, and J. Wang. Study on fluidinduced vibration power harvesting of square columns under different attack angles. Geofluids, 2017, 1-18. (2017)

[5] V. Sivadas, AM. Wickenheiser, MN. GhasemiNejhad. A study of several vortex-induced vibration techniques for piezoelectric wind energy harvesting. Active and Passive Smart Structures and Integrated Systems. (2011)

[6] HD. Akaydin, N. Elvin, Y. Andreopoulos. The performance of a self-excited fluidic energy harvester. Smart Mater. Struct. 21 025007. (2012)

[7] A. Mehmood, A. Abdelkefi, MR. Hajj, AH. Nayfeh, I. Akhtar, AO. Nuhait. Piezoelectric energy harvesting from vortex-induced vibrations of a circular cylinder. Journal of Sound and Vibration, 332(19), 4656-4667. (2013)

[8] LA. Weinstein, MR. Cacan, PM. So, PK. Wright. Vortex shedding induced energy harvesting from piezoelectric materials in heating, ventilation and air conditioning flows. Smart Mater. Struct. 21045003. (2012).

[9] HL. Dai, A. Abdelkefi, Y. Yang, L. Wang. Orientation of bluff body for designing efficient energy harvesters from vortex-induced vibrations. Applied Physics Letters, 108(5), 053902. (2016)

[10] J. Wang, S. Zhou, Z. Zhang, D. Yurchenko. Highperformance piezoelectric wind energy harvester with Y-shaped attachments. Energy Conversion and Management, 181, 645-652. (2019)

[11] J. Wang, S. Gu, C. Zhang, G. Hu, G. Chen., K. Yang, and al. Hybrid wind energy scavenging by coupling vortex-induced vibrations and galloping. Energy Conversion and Management, 213, 112835. (2020)

[12] FR. Liu, WM. Zhang, LC. Zhao, HX. Zou, T. Tan, ZK. Peng, and al. Performance enhancement of wind energy harvester utilizing wake flow induced by double upstream flat-plates. Applied Energy, 257, 114034. (2020)

[13] S. Wang, W. Liao, Z. Zhang, Y. Liao, M. K. Yan. Development of a novel non-contact piezoelectric wind energy harvester excited by vortex-induced vibration. Energy Conversion and Management, 235, 113980. (2021)
[14] A. Mehmood, AH. Nayfeh, MR. Hajj. Effects of a non-linear energy sink (NES) on vortex-induced vibrations of a circular cylinder. Nonlinear Dynamics volume 77, pages667-680. (2014)

[15] S. Leadenham, and A. Erturk. Unified nonlinear electroelastic dynamics of a bimorph piezoelectric cantilever for energy harvesting, sensing, and actuation. Nonlinear Dynamics volume 79, pages 1727-1743. (2015)

[16] A.-B. Wang, Z. Trávníček, and K.-C. Chia. On the relationship of effective Reynolds number and Strouhal number for the laminar vortex shedding of a heated circular cylinder. Physics of Fluids, 12(6), 1401-1410. (2000).

[17] S. Haque, S. Nowak, R. Callaghan, A. Mukerjee, R. Prasad, M. Rahman, and A. Mitra. A Case Study of Structural Industrial Pressure Vessel Under Wind Load. Paper presented at 2019 CIEC, New Orleans, LA. (2019)

[18] K. Yang, and al. Piezoelectric wind energy harvesting subjected to the conjunction of vortex-induced vibration and galloping: comprehensive parametric study and optimization. Smart Mater. Struct. 29 075035. (2020)

[19] M. Liu, and F. Yang. Finite element simulation of the effect of electric boundary conditions on the spherical indentation of transversely isotropic piezoelectric films. Smart Materials and Structures, 21(10), 105020. (2012) 